(Aus der psych.-neur. Klinik der R.-U. in Utrecht [Prof. Heilbronner].)

\title{
Primäres, metastasierendes Gehirncarcinom ${ }^{1}$ ).
}

\author{
Von \\ F. S. van Bouwdijk Bastiaanse, \\ Assistent der Klinik. \\ Mit 11 Textfiguren und 1 Tafel. \\ (Eingegangen am 1. Juli 1914.)
}

Primäre Gehirncarcinome gehören, im Gegensatz zu den primären benignen epithelialen Geschwülsten, die ziemlich häufig als Nebenbefund bei der Obduktion gefunden werden und nur durch Verlegen der Zirkulation der Cerebrospinalflüssigkeit klinisches Interesse darbieten, wohl zu den allergrößten Seltenheiten.

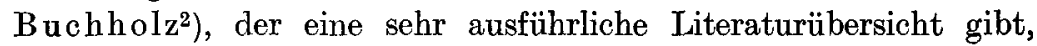
und aus Statistiken (Allen Star 600 Tumoren, Beauclair 244, Hale White von 5830 Sektionen in Gu ys Hospital 99, Klebs von 3622 Sektionen 64, Op penhei m 23), die wohl teilweise dieselben Fälle enthalten, und massenhaften kasuistischen Mitteilungen über Gehirntumoren 66 Fälle von Carcinom sammeln konnte, gibt an, daß direkt als primäre Gehirncarcinome nur die Fälle von v. Wunschheim, Coats, Späth, Gedge und Russel bezeichnet worden sind; Saxer fügt noch die Fälle von Rokitansky, Morning, Ziegler, Kaufmann und zwei von Cornil hinzu. In den letzten Jahren sind noch Mitteilungen von $\mathrm{Köl} \mathrm{pin}^{3}$ ) und von $\mathrm{Hart}^{4}$ ) hinzugekommen. Hart, der alle Mitteilungen kritisiert hat und für die Malignität infiltratives Wachstum und Metastasierung fordert, meint, daß außer seinem eigenen nur die Fälle von Cornil und $W$ unschheim als sicher primäres Carcinom aufgefaßt werden können. Im Fall von Kölpin konnte die vollständige Obduktion nicht gemacht werden, immerhin rechnet Hart auch diesen unter die primären Carcinome.

Nur in den Fällen von Spät ${ }^{5}$ ), Köl pin und Hart waren die Tumoren multipel im Gehirn, hatten also metastasiert, während Hart auch

1) Dieser Fall wurde mitgeteilt auf der Versammlung des Holländischen Psych.-Neur. Vereins in Utrecht, Dezember 1913.

2) Monatsschr. f. Psych. u. Neurol. 4, 1898.

3) Archiv f. Psych. 45.

4) Archiv f. Psych. 48.

5) Primärer multipler Epithelkrebs des Gehirns. Inaug.-Diss. München 1882. 
außerhalb des Gehirns, nämlich in den Supraclaviculardrüsen Metastasen des Adenocarcinoms gefunden hat.

Die folgende Beobachtung soll einen neuen Beitrag dazu bilden:

\section{Krankengeschichte:}

Patientin B., 57 Jahre alt. Aufgenommen 10. Juli 1913. Anamnese: Früher nie krank. Vor 8-7 Wochen plötzlich heftige Kopfschmerzen beim Saubermachen der Kirche, die Schmerzen waren so stark, daß Pat. fast nicht weiter arbeiten konnte; doch arbeitete sie noch 4 Tage, mußte dann zu Bett bleiben. Kopfschmerzen rechts sowohl wie links, im ganzen Gesicht, in der Stirn stärker als im Hinterkopf, am stärksten über der Nase und in den Ohren. Manchmal Schmerzen oben am Nacken; müdes Gefühl in den Beinen, das Gehen fiel in den ersten vier Tagen stets schwerer. Seit dem ersten Auftreten sind die immer gleichstarken Kopfschmerzen nie weggeblieben. Pat. blieb fest im Bett liegen, da die Schmerzen beim Sitzen stärker waren. In den letzten 6 Wochen schlief und a $B$ sie schlecht, es war ihr oft übel, erbrach auch einmal am Tag; oft schwindelig. Keine Sprach-, Schluck-, Gesichts- oder Gehörstörungen; nie Doppeltsehen.

Status: Pupillenreaktion-, nichts Besonderes. Feiner, nicht schneller Nystagmus beim Sehen nach links, nach rechts weniger regelmäßiger Nystagmus. Augenbewegungen maximal; koordiniert. Corneareflex links stark herabgesetzt, rechts auch nicht sehr lebhaft. Keine Adiadokokinesis. Feine Fingerbewegungen, links langsamer als rechts, der Unterschied vielleicht etwas größer als normal (lang. same Fingerbewegung nicht massal ${ }^{1}$ ). Arme und Beine motorisch nicht gestört. Zehenbewegung $\mathbf{l}=\mathrm{r}$. Tonus in allen Extremitäten gering, $\mathrm{l} .=\mathrm{r}$. Bauch-, Patellar- und Achillessehnenreflex,$+ 1 .=r$. Fußsohlenreflex links deutlich plantär, rechts keine Reaktion (durch verschiedene Untersucher wiederholt festgestellt). Der linke Arm bei Finger-Nase-Versuch und beim Zeigen mit geschlossenen Augen nach dem Hammergriff, unsicherer als der rechte Arm. Beim Emporheben und langsamen Wiederhinlegen der Beine zwischen links und rechts kein Untersehied. Kinästhesie und Gefühl für Schmerz und leises Berühren nirgends gestört. Beim Sitzen keine Rumpfataxie. Mit Rücksicht auf den Allgemeinzustand wird von Steh- und Ganguntersuchungen abgesehen. Pat. erbricht oft und klagt über starke Kopfschmerzen.

Das Gehör nahm sehr schnell ab. Bei der Aufnahme war es noch nicht sehr gestört, nach einer Woche wird rechts bei lautem Schreien a und o gehört; links bei lautem Schreien noch Zahlen. Links wird C - während 31 Sekunden gehört normal 85 Sek.; C 52 Sek. - normal 8 Sek. Rechts wird nur zwischen d 5 und f 6 gehört. Einige Tage vor der Operation war die Taubheit vollständig. Man konnte sich nur durch große aufgeschriebene Buchstaben verständlich machen. Feruch links \pm nihil, rechts ausreichend. Fundus oculi links ein dickes GefäB im umgekehrten Bild nach oben; Papille nicht ganz hell. Rechts Papille etwas trübe, gut begrenzt, Gefäße ziemlich dünn.

Psychisch war Pat. nicht sehr gestört, so daß sie selbst über die Operation entscheiden konnte; die bezüglichen Fragen wurden mit großen roten Buchstaben aufgeschrieben, ihr vorgelegt und so ihre Zustimmung erhalten. Pat. hatte vollkommene Einsicht für den Ernst ihres Zustandes. Auch die Merkfähigkeit war nicht gestört.

1) Wir unterscheiden in der Klinik: Verlangsa m ung der Fingerbewegungen (am typischsten bei Kleinhirnaffektion) und massale Fingerbewegung, die typische Störung bei der gewöhnlichen Hemiplegie.

z. f. d. ges. Neur. u. Psych, O. XXVII. 
Schon einige Tage nach dem Auftreten der Taubheit sprach Pat. agrammatisch, mit schriller, unausgeglichener Stimme.

Die Röntgenaufnahme zeigt eine normale Sella turcica; auch in Dorsoventralrichtung nichts Besonderes.

9. VIII. Neissersche Hirnpunktion in der linken Kleinhirnhemisphäre (Dr. Remeynse). Punktionsmaterial wird in Formol aufgefangen und in Paraffin eingebettet ${ }^{1}$ ). In den mit Toluidinblau gefärbten Präparaten (Tafel II, Fig. 1 und 2) befinden sich Stückchen von Kleinhirngewebe; daneben in allen Präparaten ein Netz von Fäden, die sich sehr stark färbten. Vorläufig wurden keine Zellen oder Kerne zwischen diesen Fäden gefunden. Eine dünne Lage Hühnereiweiß, gefällt mit Alkohol und mit Toluidinblau gefärbt, ergab dasselbe Netz von Fäden, so daß wohl angenommen werden kann, daß die Fäden auch aus einem eiweißartigen Stoff bestanden.

In dem Stückchen Kleinhirngewebe fand sich dieser fadenartige Stoff nirgends; er färbte sich mit Viktoriablau ebenso stark; viel weniger mit Hämatein-Eosin, auch mit der ,Dreifachfärbung“" (HämatoxylinSafranin-Pikrinsäure) wenig. Bei aufmerksamem und genauem Suchen glückte es schließlich, in einzelnen Präparaten Zellen zu finden, manchmal eine Zelle, dann wieder 6-10 zusammen. Diese Zellen waren protoplasmareich, während sich in dem Protoplasma hier und da vakuolartige Löcher befanden. In den Stückchen Kleinhirngewebe waren hier und da kleine Risse, als ob das Gewebe ödematös sei. Keine Glia wucherung, die Struktur des Kleinhirngewebes normal.

Die Diagnose war: Myxom in der linken Hemisphäre.

11. VIII. Operation (Dr. Remynse). Pat. war somnolent, hatte die letzten Tage keine Nahrung zu sich genommen.

Die linke Hemisphäre wird bloßgelegt, hier kein Tumor, wohl einige glänzende Erhebungen auf der Pia, wovon zur Untersuchung ein Stückchen entfernt wird; auch beim Einschneiden kein Tumor.

Dann wurde die rechte Hemisphäre bloßgelegt, auch hier einzelne glänzende Knötchen, aber kein größerer Tumor.

Am selben Abend Exitus.

Obduktion (Prosektor Dr. K u yer): Operationsöffnung in der Schädeldecke unter dem Sinus transverus. Die Dura ist gerade noch aufzuheben. Gyri etwas abgeplattet. Pons wird quer durchgeschnitten. Beide Kleinhirnhemisphären sind abgeplattet. Links vorn in der linken Hemisphäre des Kleinhirns befindet sich ein kleiner, nußgroßer, gelatinös aussehender Tumor (Fig. 1), circumscript, mit knotiger Ober-

1) Ausdrücklich möchte ich darauf aufmerksam machen, bei der in allen Fällen wohl nicht zu umgehenden Paraffineinbettung die Schnitte mit Eiweißglycerin (japanische Methode) aufzukleben, um zu vermeiden, daß, was bei anderen Methoden leicht geschieht, gerade die bröcklige Tumormasse bei der Bearbeitung verloren geht. 
fläche. Teilweise sitzt dieser Tumor an der Oberfläche, dringt sogar vor und hängt mit der Umgebung so wenig zusammen, daß Vorsicht geboten erscheint, damit er sich nicht aus dem umgebenden Gewebe loslöst. Augenscheinlich keine anderen Knoten in dem Cerebellum. Das Großhirn wird in frontale Schnitte zerlegt. In beiden Hemisphären finden sich in großer Anzahl ähnliche kleine Tumoren, die alle sehr oberflächlich festsitzen (Fig. 2). Die meisten sind gelatinös, einzelne sind etwas härter und sehen weiß-grau aus. Im Mark oder in den basalen Ganglien ist kein einziger Tumor gefunden worden. Bei der Sektion wurde durch den Prosektor Dr. Ku yer sehr sorgfältig nach einem

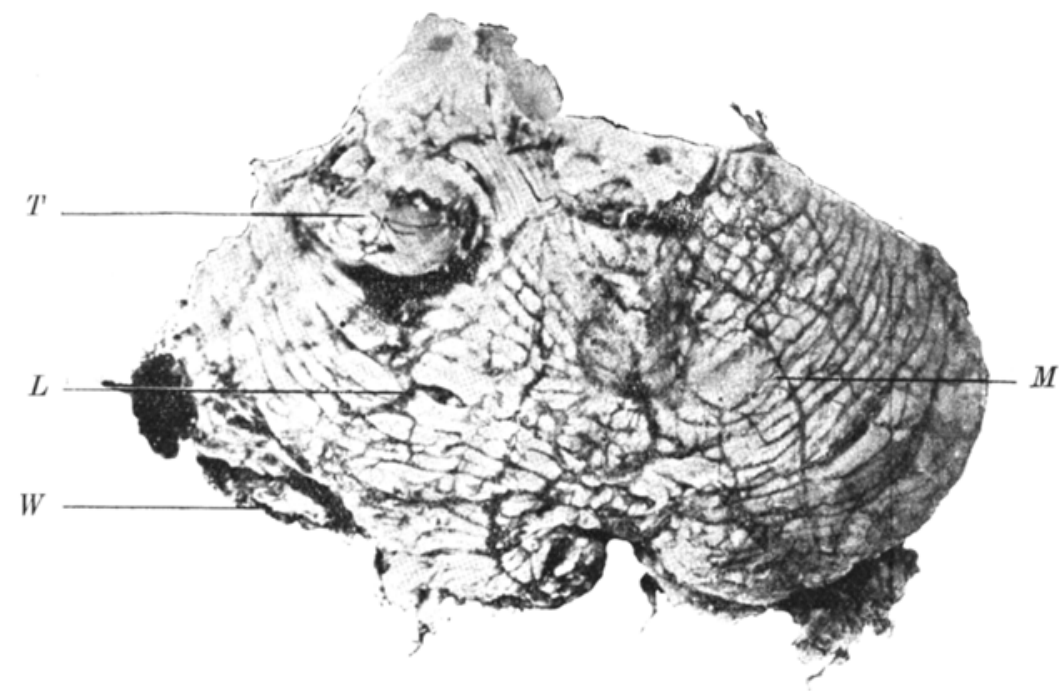

Fig. 1. Kleinhirn vor Hürtung in Formol. $T=$ Tumor. $W=$ Wunde von der Operation. $M=$ Metastase in der Pia, $L=$ Loch von der bei der Operation weggeschnittenen Metastase.

Tumor gesucht, der als primärer Ausgangspunkt dieser multiplen Hirntumoren hätte aufgefaßt werden können - ohne Erfolg. Ferner noch Bronchialpneumonie rechts und links, Paralysis cordis, Arteriosklerosis.

Das Ganze wird in Formol gehärtet. Nachdem das Präparat einige Zeit in 10proz. Formol gelegen hatte, schwoll die gelatinöse Masse außergewöhnlich stark an. Der große Tumor im Cerebellum wurde ein Konglomerat von Blasen, die sich weit über die Hirnoberfläche erhoben (vgl. Fig. 3). Aber auch die Metastasen wurden durch das Aufquellen viel deutlicher sichtbar, so daß diese noch viel zahlreicher waren, als ursprünglich vermutet wurde. Auf der Pia des Kleinhirns und in der Großhirnrinde unzählige Metastasen, von kaum sichtbaren bis haselnußgroßen. Auch jetzt zeigte sich, daß alle diese Metastasen in der Rinde 


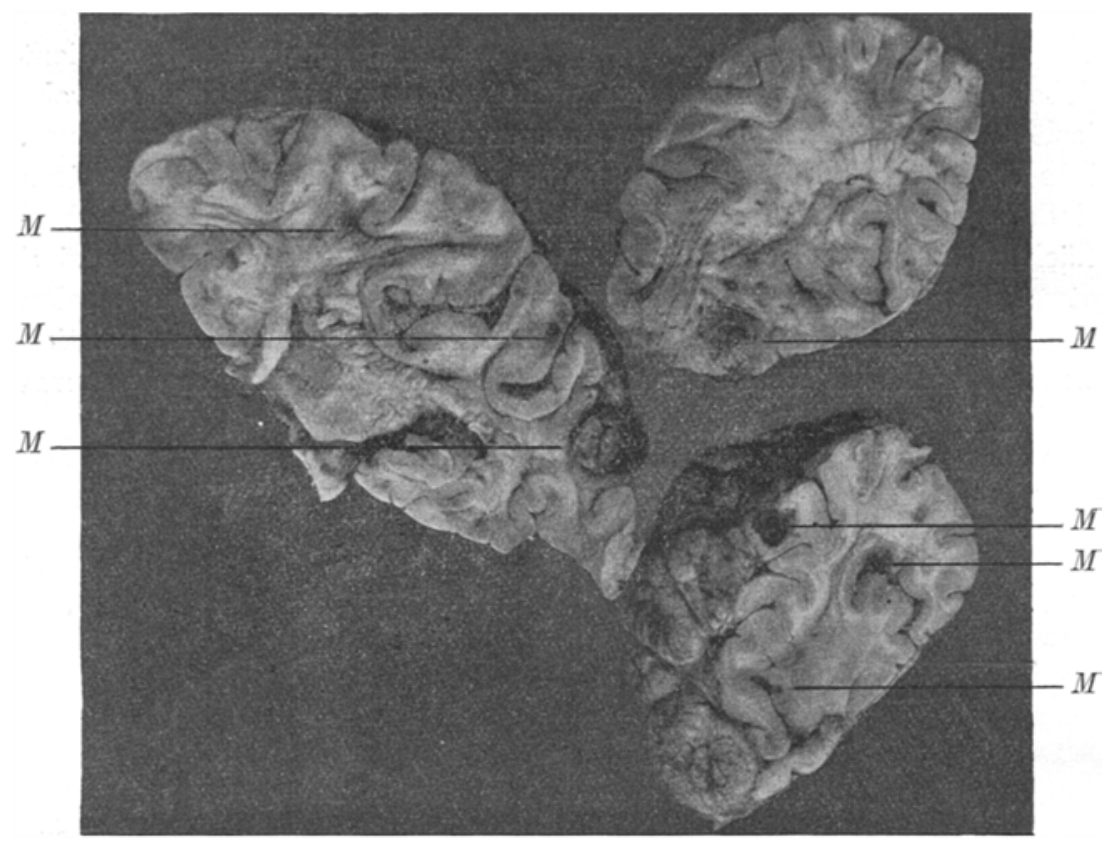

Fig. 2. Schnitte vom Großhirn. $M=$ Metastase.

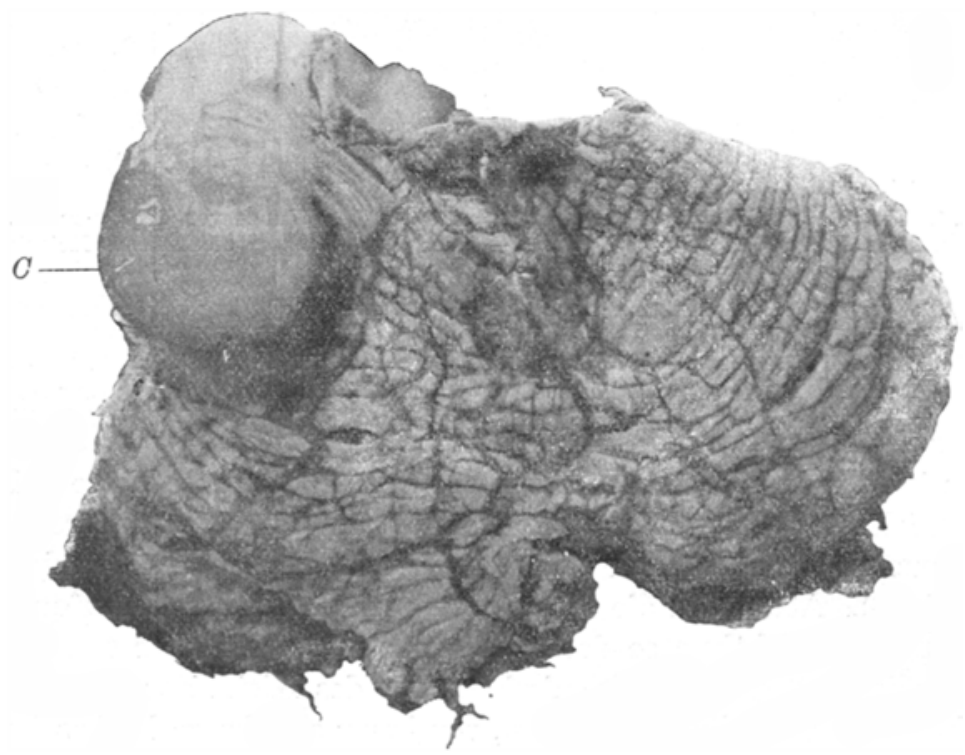

Fig. 3. Kleinhirn nach Härtung in Formol. $C=$ gequollene Cysten vom primären Tumor. 
oder der Pia saßen; bei einem einzelnen, der scheinbar noch gerade im Mark saß, ergab sich beim Ausschneiden, da $B$ er zum größten Teil in der Rinde eines tiefgehenden Sulcus festsaß. Auch die Metastasen sind gegen die Umgebung sehr scharf abgegrenzt, sie sind leicht abzulösen.

Der 4. Ventrikel hatte makroskopisch eine vollkommen normale Form, der Tumor war also nicht in die Ventrikel eingedrungen.

Mikroskopisch: Der nach der Hirnoberfläche gewandte Teil der Cystenwand des primären Tumors bestand aus einer Haut von zellarmem Bindegewebe; die nach dem Hirngewebe gerichtete Wand bestand aus einem papillösen Gewebe, dessen Papillen aus einem Bindegewebestroma, umgeben von einer Lage zum großen Teile hoher Zylinderepithelzellen (Fig. 4), zusammengesetzt war (Fig. 5). Das Stroma ist reich an Blutgefäßen und kleinzelligen Infiltrationen, während das Bindegewebe da, wo diese Infiltrationen fehlen, nichtzellenreich ist. Die Kernedes Zylinderepithels liegen alle am RandedesStromas. Die

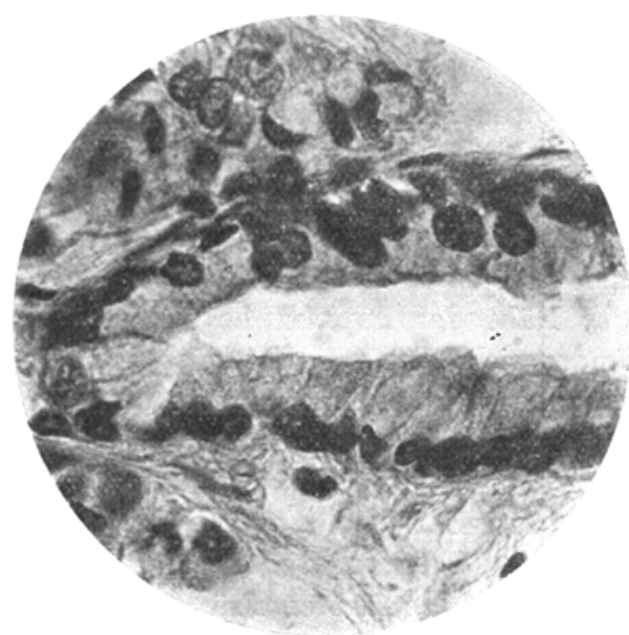

Fig. 4. Hohe Cylinderepithelzellen aus der Cystenwand von Fig. 6. Homog. Imm. Apochr. $2 \mathrm{~mm}$. Balglänge $31 \mathrm{~cm}$. Färbung: Hämatein-Eosin. Exp. $/ / 4$ Sek. Anmerkung: Alle Mikrophotos wurden mit dem großen Apparat von Zeiß gemacht.

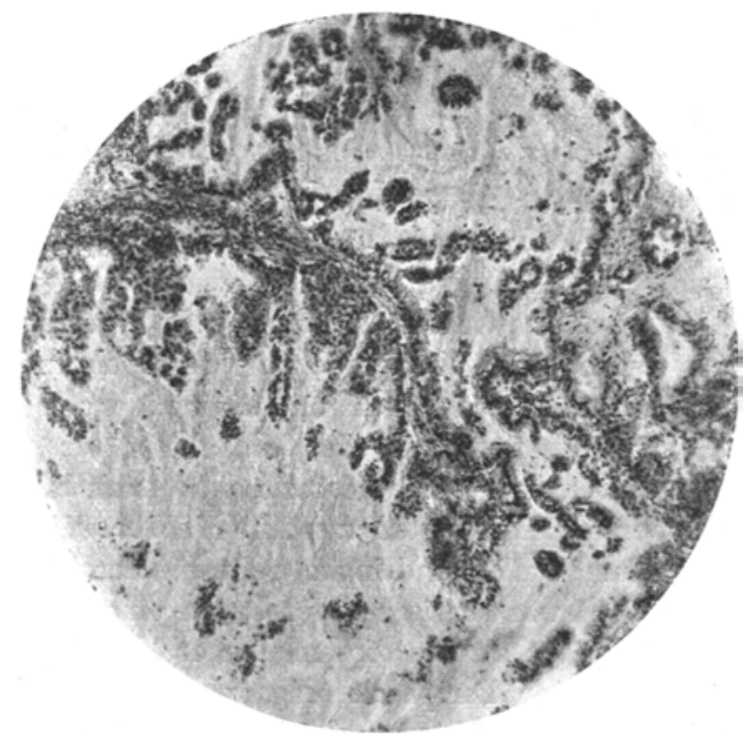

Fig. 5. Papillen aus der Cystenwand. ZeiBlinse $16 \mathrm{~mm}$. Proj. Okular $2 \mathrm{~mm}$. Balglänge $55 \mathrm{~mm}$. Exp. 1/16 Sek. Färbung: Hïmatein-Eosin. 
nach der Cyste zugekehrte Oberfläche ist rauh und es macht den Eindruck, als ob aus den Zellen etwas nach außen getreten sei. Nirgends ist diese Rauheit so, daß man mit einiger Wahrscheinlichkeit das Vorhandensein von Flimmerhaaren annehmen könnte. Die Papillen sind sehr schlank, schmal und lang; deutlich ist zu bemerken, wie an den Spitzen die Zellen abgestoßen werden. (Kerne pyknotisch, das Protoplasma färbt sich weniger, Zellgrenzen verschwinden, Epithellage ist hier und da unterbrochen, während Zellreste im Innern der Cyste in der Nähe der Papillen liegen.) An einigen Stellen der Cystenwand finden sich Klumpen Epithelzellen, deren Epithel dann mehr kubische Form hat, überall noch protoplasmareich ist und hier und da Kernteilungsfiguren aufweist.

Der Inhalt der Cyste färbt sich mit Toluidinblau stark, wenig mit Hämatein-Eosin und mit Hämatein-Safranin-Pikrinsäure. Weder in den mit Haidenhains Hämatoxylin-Eisenlack, noch in den mit Säurefuchsin gefärbten Präparaten sind Granula in den Epithelzellen zu finden. Die Epithelzellen in dem Bindegewebestroma sind scharf voneinander getrennt; die Epithelzellen stoßen mit gerader Basis an das Bindegewebe und haben nirgends fadenförmige Ausläufer. Wie zu erwarten war, ist in mit verschiedenen Gliafärbungen (Weigert, Mallory-Ribbert, Merzbacher) gefärbten Präparaten nichts von Glia gefunden worden, während von Gieson-Präparate beweisen, daß das Stroma aus Bindegewebe besteht.

Die Metastasen bestanden aus kleineren und größeren Cysten, deren Wand durch dasselbe papilläre Gewebe gebildet wurde. In der Umgebung einer großen Cyste, in dem Bindegewebestroma, liegen kleinere Cysten, die hier und da miteinander oder mit der großen Cyste zusammenfließen (Fig. 6 und 7). Die Scheidungswand wird immer dünner, schließlich kommt es zu einer Öffnung und an Stelle der Wand liegen 2 Papillen. Auch das Größerwerden des Tumors kann man am besten an den Metastasen beobachten. Fig. 6 und 7 zeigen, wie die Vergrößerung der Cyste durch Verschmelzung mit den umliegenden Cysten stattfindet. Auf Fig. 8 sieht man, wie eine Menge Metastasen in dem ödematösen Gehirngewebe in der Umgebung der großen Metastase liegen, hier das Gehirngewebe destruieren, Cysten bilden, die ihrerseits wieder mit den anderen Cysten zusammenfließen. Während also einerseits stets neue Tumorzellen eindringen, geht andererseits durch das Durchbrechen der Scheidewand zwischen den Cysten eine Menge Tumorzellen zugrunde.

Man sieht also in diesem Tumor 3 Kategorien von Zellen:

1. An der Peripherie ungeordnete protoplasmaarme Zellenhaufen mit reichlichen Mitosen als Ausdruck starker Wucherung,

2. regelmäßig angeordnete hochzylindrische, in einfacher Lage dem Stroma aufsitzende Epithelzellen, 


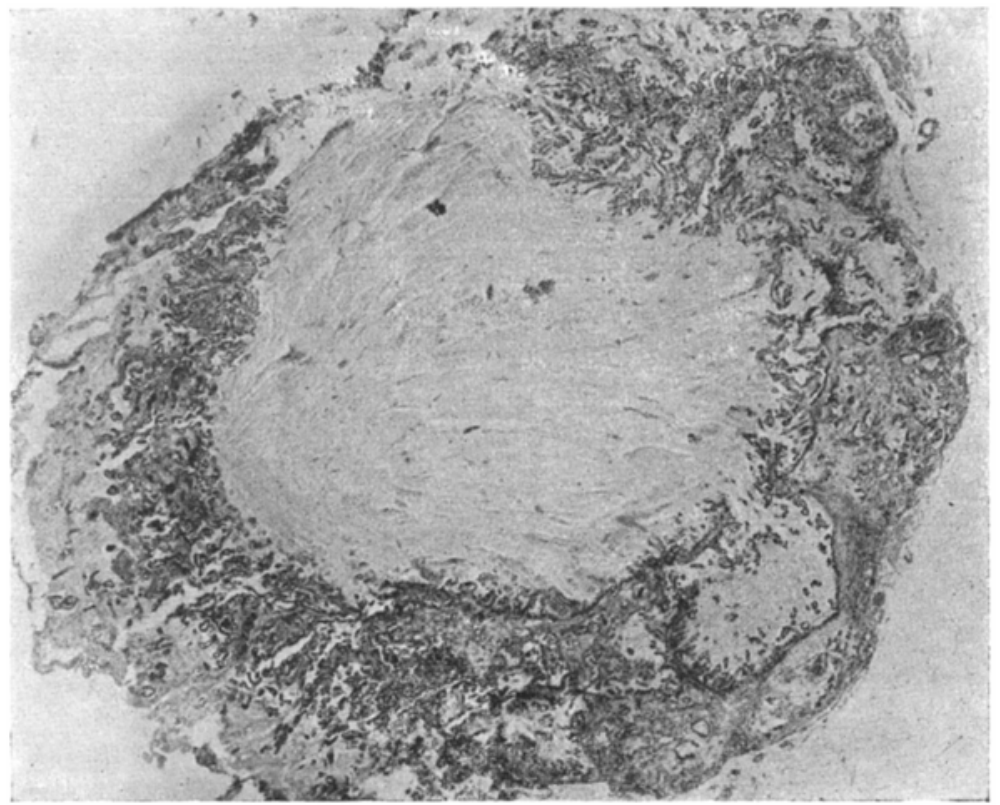

Fig. 6. Metastase im Großhirn. Cyste mit kleineren Cystesis der Umgebung. Mikroplanar $35 \mathrm{~mm}$. Balglänge $31 \mathrm{~cm}$. Exp. 1/25 Sek. Fărbung: Hămatein-Eosin

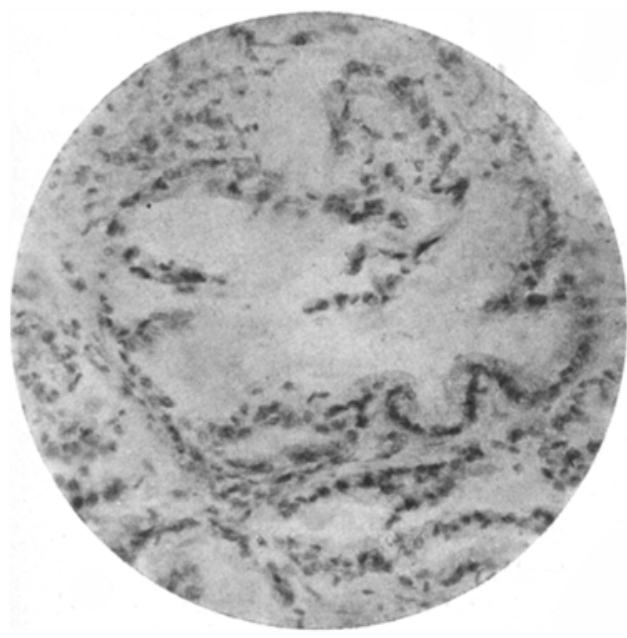

ig. 7. Kleine Cyste aus der Metastase abgebildet I Fig. 6. Zeiß-Linse obj. $4 \mathrm{~mm}$. Proj. Okular $2 \mathrm{~mm}$. Balglänge $41 \mathrm{~cm}$. Exp. 1/2 Sek. Färbung: Hämatein-Eosin.

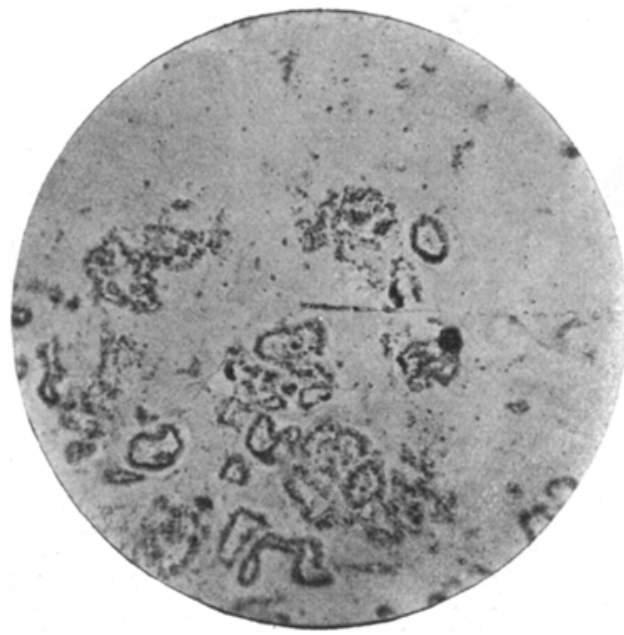

Fig. 8. Kleine Metastasen in der Umgebung einer großen. Zeiß-Linse $16 \mathrm{~mm}$. Proj. Okular $2 \mathrm{~mm}$. Balglänge $48 \mathrm{~cm}$. Exp. $1 / 16$ Sek. Färbung: Hzmatein-Eosin. 
3. Zellen mit Zeichen des Zerfalls (schlecht färbbarem Protoplasma, pyknotischen Kernen), zum Teil in der Cystenflüssigkeit liegend.

Auch bei den kleineren Cysten ist überall das Protoplasma der Epithelzellen nach dem Cystenzentrum, die Kerne nach dem Bindegewebestroma zugekehrt.

Am Rand der großen Cysten finden sich sehr viele in kleine Kreise gruppierte Epithelzellen, deren scheinbarer Hohlraum im Zentrum ungefähr dieselbe Größe hat wie die kleinen Cysten. Sie unterscheiden sich jedoch von diesen: 1. sind die kleinen Cysten von Bindegewebe umgeben, die kreisförmig gruppierten Epithelzellen von Cysteninhalt,

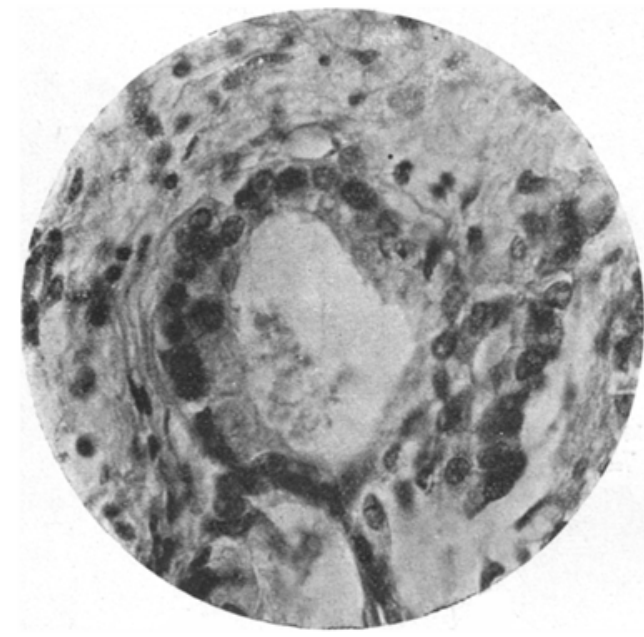

lig. 9. Kleine Cyste. Hom. Imm, Apochr. $2 \mathrm{~mm}$. lalglänge $30 \mathrm{~cm}$. Proj. Okular $1 \mathrm{~mm}$. Exp. 1/a Sek. Farbung: Hämatela-Eosin.

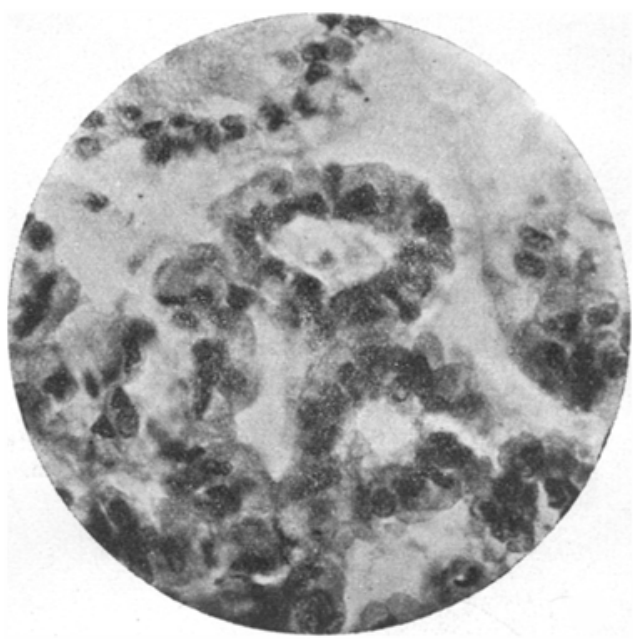

Fig. 10. Querdurchschnitt von Papillen. Hom. Imm. Apochr. $2 \mathrm{~mm}$. Balglänge $32 \mathrm{~cm}$. Proj. Okular $2 \mathrm{~mm}$. Exp. $1 / \mathrm{g}$ Sek. Färbung: Hämatein-Eosin.

2. liegen die Kerne der die Cyste begrenzenden Epithelzellen nach der Mitte zu (Fig. 9), die der kreisförmig gruppierten nach der Peripherie zu (Fig. 10). Diese letzteren sind also wohl als Querdurchschnitte der in der Cystenflüssigkeit schwimmenden Papillen aufzufassen. In einigen Zentren findet man auch hier und da einen Bindegewebekern.

Außer den großen, makroskopisch schon wahrnehmbaren Metastasen finden sich in Präparaten von makroskopisch normaler Pia noch vielfach kleinere Zellenanhäufungen, die nur in der unmittelbaren Nähe des großen Tumors oder der großen Metastasen sich flach ausgebreitet haben, meistens jedoch den Charakter von kleinen Tumoren behalten haben. Wie Fig. 10 zeigt, liegen in der Umgebung der größeren Metastasen in der Hirnsubstanz unregelmäßig verstreut ödematös aussehende Gruppen von 5-10 Zellen. 
Eine 4. Form von Metastasierung zeigt Tafel II, Fig. 3. Diese Gefäße liegen inmitten von Gliagewebe in der Rinde, ungefähr einen halben Zentimeter von einer größeren Metastase entfernt.

Außerdem sind sehr viele Gefäße mit Zellen infiltriert, die wenig Protoplasma enthalten und nur in der Umgebung des Tumors oder der größeren Metastasen zu finden sind. Es läßt sich nicht sicher feststellen, ob dies sehr junge Tumorzellen sind.

Das Epithel der Ventrikelwände, auch das des Plexus chorioidei des 3. und 4. Ventrikels ist von Tumorgewebe vollständig frei.

Weder in der Umgebung des Tumors noch der Metastasen ist etwas von Gliawucherung zu finden (Toluidinblau, Mallory-Ribbert, Weigert, Merzbacher).

Klinisch bietet der Fall nichts Besonderes, um so mehr, da der desolate Allgemeinzustand und die bald aufgetretene totale Taubheit, die eine Verständigung nur noch auf schriftlichem Wege möglich machte, eine genaue Untersuchung ausschlossen. (Die beiderseitige totale Taubheit ist übrigens auch durch den Sektionsbefund nicht geklärt, wenn man sich nicht mit der Druckwirkung auch auf den Acusticus r'er anderen Seite begnügt.)

Nicht einmal die Tumordiagnose war angesichts der fehlenden Augenhintergrunderscheinungen mit Sicherheit zu stellen. Erbrechen und Kopfschmerzen waren immerhin verdächtig genug und der weln auch geringe $\mathrm{N}$ ystagmus nach links, das Fehlen des Cornealreflexes links, die schwere Beeinträchtigung des Gehörs (und die leichte Beeinträchtigung der Fingerbewegung links) ergaben doch genügenden Verdacht auf die linke Kleinhirnhemisphäre, um die Punktion dieser Gegend gerechtfertigt erscheinen zu lassen, lie auch sofort Tumorgewebe ergab. Histologisch ergab sich ein epithelialer Tumor, der infiltrativ wächst und Metastasen hervorruft, ein Carcinom also. Einer der Tumoren, von denen Borst ${ }^{1}$ ) sagt: „Eine etwas abweichende Form von Papillom (Peritheliom), die nach v. Rindfleisch häufiger vorkommt, früher aber vielfach als Myxom angesprochen wurde, zeichnet sich dadurch aus, da $\beta$ die sehr langen und wohlgebildeten Zylinierzellen der Papillen, wie an der Oberfläche einer Schleimhaut, Schicht auf Schicht einen zähen, glasigen Schleim absondern (Papilloma myxomatodes); die Geschwulst kommt auch multipel am Gehirn vor und entwickelt sich ebenfalls gerne von den Plexus chorioidei", erinnert in so vieler Hinsicht an unseren Tumor, daß ich nicht wagte, die Differentialdiagnose zu stellen, sondern einige Präparate an Prof. Borst sandte, der dic Güte hatte, sie zu untersuchen. Aus seiner Antwort zitiere ich wörtlich: „Es liegt ein zweifellos epithelialer Tumor vor" und ferner: „Ein Peritheliom liegt sicher nicht vor."

1) Die Lehre von den Geschwülsten. 
Das Fehlen von deutlicher Stauungspapille ist bei einem derartig großen Tumor in der hinteren Schädelgrube und einer so großen Anzahl Metastasen jedenfalls auffallend. Ich glaube, daß dies mit der geringen Druckerhöhung, die sich während der Operation zeigte und sich auch durch die geringe reaktive Gliawucherung äußerte, in eine Reihe zu stellen ist. Auch Hart, Kölpin und Buchholz (bei letzterem fehlte in einem Fall von multiplem sekundärem Hirncarcinom Stauungspapille) weisen auf die geringe Gliawucherung und andere Drucksymptome um clie Tumoren hin. Kölpin sagt: „Man muß. also wohl annehmen, daß Wachstum der Neubildung und Zugrundegehen von Hirn-

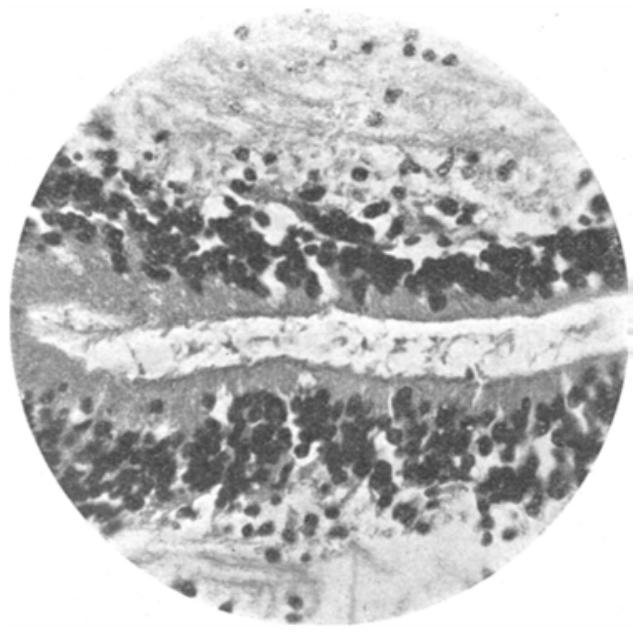

Fig. 11. Ventrikelwand von einem Foetus von $2 \mathrm{~cm}$ Länge. Schnit zur Höhe der Augen. Immen. 2 mm. Proj. Okular $2 \mathrm{~mm}$. Balglänge $30 \mathrm{~cm}$. Exp. ${ }^{4}$ Sek. Färbung: Hämatein-Eosin. substanz annähernd gleichen Schritt halten, sich quantitativ annähernd entsprechen ${ }^{1}$ ). "

Unaufgeklärt bleibt trotz allem Suchen der Ausgang des Tumors; auch mikroskopisch zeigte sich die Wand des 4. Ventrikels, inklusive des Plexus ganz frei von Tumorzellen.

Trotzdem wird, wenn man die Tumorzellen mit dem Ependym eines Foetus von $\pm 2 \mathrm{~cm}$ Länge (Fig. 11) vergleicht, die Utbereinstimmung beider Zellformen auffallen. Das Ependym des jungen Foetus hat noch die hohe Zylinderepithelform, die erst später in eine viel plattere Form übergeht. Man könnte also mit Cohnheim annehmen, daß derartige Zellen versprengt sind, sich dann nicht weiter differenzieren und, auch wenn sie zu wuchern anfangen, auf dieser tiefen Entwicklungsstufe stehenbleiben. Auch die Pyramidenzellen des fötalen Ependyms zeigen dieselbe rauhe Oberfläche, als ob etwas ausgeschieden würde. Ob diese rauhe Oberfläche durch ein von diesen Zellen ausgeschiedenes Sekret, ob durch das Zerreißen von vielleicht vorhandenen Flimmerhaaren infolge der Bearbeitung entsteht, wage ich nicht zu entscheiden. (Fuchs, Cavazzani, Yoshimurau. a. sehen die Ependymzellen als Drüsenepithel an ${ }^{2}$ ), während Mestrezat ${ }^{3}$ ) ebenso brstimmt.

1) 1. c. S. 602. - $\left.{ }^{2}\right)$ Vgl. Victor Kafka, Die Cerebrospinalflüssigkeit. Zeitschr. f. d. ges. Neur. u. Psych. Ref. u. Erg. 6, Heft 5.

3) Le liquide Céphalo-Rachidien S. 184. 
meint, daß die Cerebrospinalflüssigkeit ,un liquide dialysé " ist und von ,cellules dyalisantes" spricht.)

Besonders Bittorf ${ }^{1}$ ) weist auf den entwicklungsgeschichtlichen Unterschied hin, der zwischen dem von den beiden Seitenhälften stammenden Epithel und dem der Boden- und Deckplatte besteht. Aus dem ersteren bilden sich Spongioblasten und Ependymzellen, die beide Gliagewebe hervorrufen; die Boden- und Deckplatte bildet nur eine einfache Epithellage. Die von den Seitenhälften ausgehenden Tumoren zeigten stets Neigung zur Biliung von Gliagewebe, im Gegensatz zu denen der Boden- und Deckplatte, die rein epithelial blieben.

Es scheint mir am wahrscheinlichsten, daß auch in unserm Fall der Tumor von Elementen des Plexusepithels (Deckplatte), wenn auch von einem versprengten Keim, ausgeht.

Kölpin glaubt, daß in seinem Fall (bei dem durch unvollständige Sektion nicht erwiesen war, daß der Hirntumor primär war) das nicht mit dem Ventrikelependym im Zusammenhang Stehen des Tumors und das Nichtwachsen des Tumors in der Ventrikelhöhle gegen das primäre Bestehen des Tumors spricht. Auch in urserm Fall fehlen diese beiden Umstände. Obgleich der pathologische Anatom sehr genau nach Gewebe eines primären Tumors gesucht hat und alle Organe untersucht hat, glaube ich doch, daß die Möglichkeit besteht, ein derartiges Gewebe zu übersehen.

Auffallend ist, daß die Epithelzellen bei ihrer Wucherung und Metastasierung überall durch Bindegewebe gefolgt werden, während das Gliagewebe sogar keine starke reaktive Wucherung zeigt.

Die Metastasen sitzen ausschließlich in der Rinde und nur mikroskopisch (Fig. 13) zeigt sich, daß einzelne Tumorzellen auch in das Mark vordringen.

Buchholz ${ }^{2}$ ), der eine Abbildung eines mit Tumorzellen infiltrierten Gefäßes zeigt, sagt über die Metastasierung (S. 204): „Daß übrigers dieser Modus der Verbreitung des Carcinoms der einzige ist, wage ich nicht zu behaupten; ich muß sogar annehmen, daß vielfach auch auf anderen Wegen innerhalb der feinen Lymphbahnen Keime verschleppt sein können; ich habe wenigstens nicht immer in der Mitte der kleinsten Tumoren ein Gefäß auffinden können." Es scheint mir in unserm Fall ziemlich sicher, daß die Metastasierung hauptsächlich durch die Cerebrospinalflüssigkeit stattgefunden hat; nur diese ist imstande, die Tumorzellen von der Kleinhirnoberfläche über die ganze Hirnoberfläche zu verbreiten. Der Stromrichtung der Cerebrospinalflüssigkeit ist es vielleicht auch zuzuschreiben, daß in den Ventrikelwänden keine Metastasen gefunden wurden. Fig. 10 zeigt eine zweite Metastasenform,

1) Zieglers Beiträge 'z. allg̣. Path. u. pathol. Anat. 35.

2) l. c. 
108 F. S. van Bouwdijk Bastiaanse: Primäres, metastasierendes Gehirncarcinom.

hier liegen um die Hauptmetastase kleinere in dem Hirngewebe verstreut. Die Tumorzellen müssen hier durch die kleineren Lymphbahnen verbreitet sein.

Die 3. Form (Fig. 13) ist längs der perivasculären Räume.

\section{Zusammenfassung:}

Durch die Neissersche Hirnpunktion zeigte sich bei einem Pat. mit Symptomen von Kleinhirnläsion, daß in der linken Kleinhirnhemisphäre ein schleimreicher Tumor saß, der bei der Operation nicht erreicht wurde.

Bei der Obduktion und der mikroskopischen Untersuchung stellte sich dieser Tumor als ein primäres Carcinom mit zahlreichen Metastasen in Pia und Rinde des Großhirns heraus.

Die Tumorzellen sind Drüsenepithelzellen und zeigen viel Ähnlichkeit mit den primitiven Ependymzellen des Foetus. Der Tumor ist jedoch nicht von dem die Ventrikel begrenzenden Ependym ausgegangen.

Die Metastasierung hat auf dreierlei Weise stattgefunden: 1. Verbreitung durch die Cerebrospinalflüssigkeit in der Richtung des Stroms: 2. durch die kleinen Lymphbahnen; 3. durch die Lymphbahnen längs der Blutgefäße (perivasculäre Rüume). 